\title{
Naturalness in Nuclear Effective Field Theories
}

\author{
U. van Kolck \\ Institut de Physique Nucléaire, CNRS/IN2P3, \\ Université Paris-Sud, Université Paris-Saclay, \\ 91406 Orsay, France \\ and \\ Department of Physics, University of Arizona, \\ Tucson, AZ 85721, USA
}

March 24, 2020

\begin{abstract}
Nuclear effective field theories (EFTs) have been developed over the last quartercentury with considerable impact on the description of light and even medium-mass nuclei. At the core of any EFT is a systematic expansion of observables, which is usually obtained from a rule based on an assumption of naturalness. I discuss naturalness in the context of the relatively weak binding of nuclei, where discrete scale invariance plays a role in the emergence of complexity.
\end{abstract}




\section{Introduction}

In particle physics and cosmology, one most frequently hears about the concept of naturalness in connection to problems with the Standard Model (SM) [1]: strong CP, gauge hierarchy, cosmological constant. It is easy to forget that naturalness is a cornerstone for the paradigm to understand the successes of quantum field theory across mass scales, effective field theories (EFTs) [2]. The SM is but one example of an EFT, one in which little information is known about subleading interactions. My goal here is to discuss some aspects of naturalness in nuclear EFTs, where the weak binding of nuclei is also usually seen as a naturalness problem, but subleading interactions are important and assuming some version of naturalness is crucial for predictions.

Nature is organized as a tower of EFTs 1 , each with at least two mass scales: the scale $M_{\text {lo }}$ we probe with reactions where the typical external momentum $Q \sim M_{\mathrm{lo}}$, and the breakdown scale $2 M_{\mathrm{hi}}$ where a reorganization of the theory (new degrees of freedom, reordering of interactions) is needed. In an EFT, all interactions among the relevant degrees of freedom are included which are allowed by symmetries, since even if an allowed interaction were magically absent at one scale it would still be present at other scales. The only way to make predictions is to first make an assumption about the magnitudes of masses and interaction strengths. Naturalness, in the form of an expectation about the effects of an EFT parameter on observables, offers a rule to infer the hierarchy of interactions ("power counting"). One usually speaks of fine tuning when naturalness expectations are not fulfilled and yet no new symmetry is identified. Naturalness considerations are not idle, as when naturalness fails for one observable we need to determine the extent of power-counting revision.

The scarcity of data challenging the SM is probably the engine that drives the emphasis on naturalness problems. In contrast, data abound in nuclear physics, which has proven to be fertile ground for EFTs (see Ref. [3] for a recent comprehensive review). From early on, nuclear theory has been a constant struggle to explain the many regularities seen among nuclear properties while facing severe renormalization difficulties. (For a short history, see Ref. [4].) There has always been a feeling, although it is hard to trace its origins, that "nuclear physics is fine tuned". EFT naturalness provides a framework to address this issue.

It is in fact hard to go anywhere with nuclear EFTs without guidance from naturalness. No nuclear EFT can go beyond the scale associated with nonperturbative effects in Quantum Chromodynamics (QCD), $M_{\mathrm{QCD}} \sim 1 \mathrm{GeV}$, which sets the scales for hadronic masses such as the nucleon mass $m_{N} \simeq 940 \mathrm{MeV}$. Ground states in heavy nuclei have an approximately constant binding energy per nucleon $B_{A} / A$, which we can associate with

\footnotetext{
${ }^{1}$ Just so the true believers burn me at the stake for the right reason: I ain't saying the tower never ends, only that we'll not know for sure.

${ }^{2}$ In the particle physics literature, where dimensional regularization is almost exclusively used, the physical breakdown scale is normally referred to as the "cutoff" of the theory. Unfortunately dimensional regularization is not well adapted to nonperturbative problems where loops do not factorize, as in nuclear physics (except for a very specific situation mentioned below). Here I reserve "cutoff" to the arbitrary momentum (or coordinate) cutoff introduced by the regularization procedure.
} 
a binding momentum $3 \kappa_{A} \sim \sqrt{2 m_{N} B_{A} / A}$. For the alpha particle, frequently considered as a light representative of typical nuclei, $\kappa_{4} \simeq 100 \mathrm{MeV}$. While these specific estimates for $M_{\mathrm{QCD}}$ and $\kappa_{A}$ can easily be wrong by factors of $\mathcal{O}(1)$, it is well recognized that the bane of nuclear physics is a limited separation of scales. In contrast with atoms where the Coulomb interaction is clearly dominant and effects from other electromagnetic forces are very small, in nuclear physics even the identification of leading interactions is challenging.

Much nuclear EFT work has been carried out as an uncritical application of "naive dimensional analysis" [5, 6, 7, 8, As I discuss in Sec. 2, this is a rule based on the sensitivity of loop diagrams to high-momentum physics, which applies to a large class of EFTs where all interactions are perturbative. Naive dimensional analysis frames, for example, the SM naturalness debate. But purely perturbative nuclear physics is not, else there would be no nuclei. The implications of renormalization in a nonperturbative context are perhaps the most distinctive feature of nuclear EFTs - for a review, see Ref. [9].

A characteristic feature of nuclear physics is that nucleons are heavy (compared to their binding momenta) and stable (or nearly so). Loops are sensitive to $M_{\text {hi }}$ in a different way than in most known EFTs [10, 11, 12, 13, 14, 15]. The appropriate modification of naive dimensional analysis is introduced in Sec. 3, and corroborated by specific examples. The idea that nuclear physics is fine tuned might be traced back to the fact that, even with such modification, light nuclei are unnaturally shallow.

In Sect. 4 I argue that this unnaturalness can be explained away, at least for light nuclei, by a symmetry — discrete scale invariance — whose importance has not been fully appreciated until recently [16, 17, 18, 19, 20]. Discrete scale invariance emerges within the nuclear EFT designed to deal with light nuclei, Pionless EFT, in the unitarity limit where two-body binding energies vanish. This symmetry allows for growing complexity as $A$ increases, including some threshold coincidences that one would have thought arise from delicate fine tuning.

While it is not obvious that this description can be extended to heavier nuclei, if it does one could say nuclear physics is technically natural. Some unresolved issues with this conjecture are present in Sec. [5] in guise of a conclusion.

These ideas have been explored separately before and are of course known to be related. My intention in bringing them together here is not to contribute to the history of the concept of naturalness and to the contemporary preoccupation with the gauge hierarchy problem. Many of the interesting philosophical underpinnings of naturalness have been examined in, for example, Refs. [21, 22, 23, 24, 25, 26, 27, 28, 29, 30]. My goal is instead to highlight the connection between the general ideas that permeate these discussions and a specific situation where naturalness (or lack thereof) has very observable consequences.

\footnotetext{
${ }^{3}$ This estimate gives the correct position of the $T$-matrix pole for $A=2$ and ensures that all nucleons contribute equally to the binding energy when $A \gg 2$.
} 


\section{Enunciating naturalness}

The idea that "fundamental" parameters should not differ significantly in magnitude has been around for a long time, being expressed in a particularly clear form by Dirac [31. In an EFT, fundamental parameters are replaced in an effective Lagrangian by an infinite number of interaction strengths, known as low-energy constants (LECs) or Wilson coefficients. These parameters are in general dependent on the regulator one introduces to make sense of quantum corrections; they are "bare", unobservable parameters. In observables, they always appear together with the high-momentum components of quantum corrections, which also depend on the regulator, in what are frequently referred to as "renormalized" parameters. EFT is all about observables, and when effective-field theorists talk about naturalness, they are thinking about the size of the renormalized LEC 4 . More precisely, naturalness concerns the effect the finite part of a LEC has on observables.

The simplest version of naturalness is probably plain dimensional analysis, where any LEC associated with an operator of mass dimension $D$ appearing in the Lagrangian is assumed to be

$$
c_{D}=\mathcal{O}\left(M_{\mathrm{hi}}^{4-D}\right) .
$$

While frequently this is enough for rough estimates, there are many instances where it is not. We would like to account for possible small coupling constants. And, even when the theory is strongly coupled, extra factors of $4 \pi$ can be essential in providing a low-energy scale $M_{\mathrm{lo}}$.

As an example relevant to nuclear physics, let us consider two-flavor Chiral EFT $(\chi \mathrm{EFT})\left[2\right.$, 32] - the EFT of QCD where $M_{\mathrm{hi}}=\mathcal{O}\left(M_{\mathrm{QCD}}\right)$. It includes an isotriplet of pions $\pi$ with a root-mean-square mass $m_{\pi} \simeq 137.3 \mathrm{MeV}$ and charge-neutral squaredmass splitting $\delta m_{\pi}^{2} \equiv m_{\pi^{ \pm}}^{2}-m_{\pi^{0}}^{2} \simeq(35.5 \mathrm{MeV})^{2}$, and, because they are (at least, nearly) stable, an isodoublet of nucleons $N$ with average mass $m_{N}=\left(m_{p}+m_{n}\right) / 2 \simeq 938.9 \mathrm{MeV}$ and neutron-proton mass splitting $\delta m_{N} \equiv m_{n}-m_{p} \simeq 1.3 \mathrm{MeV}$. With $\vec{\sigma}(\boldsymbol{\tau})$ denoting the Pauli matrices in spin (isospin) space, the Lagrangian can be written

$$
\begin{aligned}
\mathcal{L}_{\chi \mathrm{EFT}}= & \frac{1}{2}\left[\left(\partial_{0} \boldsymbol{\pi}\right)^{2}-(\vec{\nabla} \boldsymbol{\pi})^{2}-m_{\pi}^{2} \boldsymbol{\pi}^{2}-\delta m_{\pi}^{2}\left(\boldsymbol{\pi}^{2}-\pi_{3}^{2}\right)\right] \\
& +N^{\dagger}\left(i \partial_{0}+\frac{\vec{\nabla}^{2}}{2 m_{N}}+\frac{\delta m_{N}}{2} \tau_{3}\right) N+\frac{g_{A}}{2 f_{\pi}} N^{\dagger} \boldsymbol{\tau} \vec{\sigma} N \cdot \vec{\nabla} \boldsymbol{\pi}+\ldots,
\end{aligned}
$$

where $f_{\pi} \simeq 92 \mathrm{MeV}$ is the pion decay constant and $g_{A} \simeq 1.27$ is the nucleon axial coupling. The "..." represent terms with more fields and derivatives, as well as further isospin-breaking and weaker interactions. Plain dimensional analysis gives

$$
m_{\pi}^{2} \sim\left|\delta m_{\pi}^{2}\right|=\mathcal{O}\left(M_{\mathrm{QCD}}^{2}\right), \quad m_{N} \sim\left|\delta m_{N}\right|=\mathcal{O}\left(M_{\mathrm{QCD}}\right), \quad\left|g_{A}\right|=\mathcal{O}\left(f_{\pi} / M_{\mathrm{QCD}}\right),
$$

\footnotetext{
${ }^{4}$ When nonperturbative physics is involved, the calculation of quantum corrections is often limited to numerics and one cannot write a simple analytical formula for the renormalized LEC. In nuclear physics, this limitation causes an inordinate amount of confusion.
} 
which would suggest that at least $m_{\pi}, \delta m_{\pi}^{2}, \delta m_{N}$, and $g_{A}^{-1}$ are observed to be unnaturally small, an unlikely scenario. Clearly we should be able to do better. The existence of a gap between $f_{\pi}$ and the masses of most hadrons set by $M_{\mathrm{QCD}}$ means that, once a better guess is employed for what is natural, we should see $\chi \mathrm{EFT}$ as an EFT where $M_{\mathrm{lo}}=\mathcal{O}\left(f_{\pi}\right)$. In addition, other low-energy scales are present in the form of $m_{\pi}, \delta m_{\pi}^{2}$, and $\delta m_{N}$.

More generally, the issue is that LECs can also depend on the ratio $M_{\mathrm{lo}} / M_{\mathrm{hi}}$, since both scales emerge from the same theory at momenta above $M_{\text {hi }}$. Two essential ideas are:

- As stressed by Veltman [33], we must consider renormalization explicitly and use loop corrections to estimate the importance of a LEC. The actual values of loop integrals depend on the regulator parameter $\Lambda$ that we introduce to cut large momenta off. Once $\Lambda \gtrsim M_{\mathrm{hi}}$ there is no hope that we can account for this physics in detail. But this is also physics that can be mocked up by short-range interactions, and it is only the combination of bare LECs and high momentum in loops that enters observables. Renormalization is the demand that the $\Lambda$ dependence in the bare LECs cancels out that from the loops, no matter how large $\Lambda$ is. But the dependence on $\Lambda$ from the loops indicates the sensitivity of the observable to highmomentum physics that enters at $M_{\mathrm{hi}}$. Thus, barring cancelations in this physics, the replacement $\Lambda \rightarrow M_{\text {hi }}$ gives an indication of the natural size of the observable, controlled by the finite part of the corresponding LEC. (For a clear discussion in the context of the cosmological constant, see Ref. [34].) This requirement ends up bringing to Eq. (11) additional factors of $4 \pi$, arising from angular integration.

- 't Hooft [35] pointed out that small LECs are unlikely to arise in the EFT from cancelations among parameters in the underlying theory, and are expected instead to come from symmetries. An exact symmetry will guarantee that an infinite number of otherwise possible LECs vanish, as do loop corrections to their tree-level values (when a symmetry-preserving regulator is used). An approximate symmetry will allow both bare LECs and non-vanishing loop corrections, but they will be suppressed by powers of the small symmetry-breaking parameters: the LECs are "protected" from receiving large corrections. This idea is frequently referred to as "technical" naturalness. Equation (11) should be modified to account for parameters that break symmetries gently.

In perturbation theory, these two ideas are incorporated in naive 5 dimensional analysis (NDA) [5, 6, 7, 8]. Perhaps the simplest version is that of Weinberg's [7], where the LEC of an operator involving $N$ fields is natural if

$$
c_{D N}=\mathcal{O}\left((4 \pi)^{N-2} M_{\mathrm{hi}}^{4-D} c_{D N}^{\mathrm{red}}\right)
$$

in terms of the dimensionless "reduced" $\operatorname{LEC} c_{D N}^{\mathrm{red}}$. When the underlying theory has no small parameters, $c_{D N}^{\text {red }}=\mathcal{O}(1)$. When it does, the size of the reduced LEC is set by the minimum powers of the (small) reduced parameters associated with the underlying operators needed to generate the corresponding operator in the EFT. We can now schematically

\footnotetext{
5 "Naive" perhaps due to the modesty of the authors of Ref. [5].
} 
write a general term in an effective Lagrangian containing $b$ (relativistic) bosonic fields $\phi$, $f$ fermionic fields $\psi$, and $d$ derivatives as [5, 6, 8]

$$
\mathcal{L}_{\mathrm{EFT}} \sim(4 \pi)^{-2} M_{\mathrm{hi}}^{4} c_{D N}^{\mathrm{red}}\left(\frac{\partial}{M_{\mathrm{hi}}}\right)^{d}\left(\frac{4 \pi \phi}{M_{\mathrm{hi}}}\right)^{b}\left(\frac{(4 \pi)^{2} \bar{\psi} \psi}{M_{\mathrm{hi}}^{3}}\right)^{f / 2}
$$

using $N=b+f$ and $D=b+3 f / 2+d$. Compared to plain dimensional analysis, there are extra factors of $4 \pi$ and, per technical naturalness, $c_{D N}^{\text {red }}$.

The improvement over plain dimensional analysis is clear in $\chi \mathrm{EFT}$. The underlying theory is QCD with additional electromagnetic interactions given by QED and weaker interactions given by Fermi theory. For two flavors - up and down quarks of masses $m_{u}$ and $m_{d}$ - the small parameters are the reduced average quark mass $\hat{m}^{\text {red }}=\hat{m} / M_{\mathrm{QCD}} \equiv$ $\left(m_{u}+m_{d}\right) / 2 M_{\mathrm{QCD}} \sim 0.003$, the reduced down-up quark-mass difference $(\epsilon \hat{m})^{\text {red }}=\left(m_{d}-\right.$ $\left.m_{u}\right) / 2 M_{\mathrm{QCD}} \sim \hat{m}^{\text {red }} / 3$, the reduced electromagnetic coupling $e^{\text {red }}=\sqrt{\alpha_{e} / 4 \pi} \sim 0.02$, and reduced parameters associated with weaker interactions. The smallness of the quark masses is technically natural because when they are zero the theory has a chiral symmetry of independent rotations of left- and right-handed quarks. In two-flavor QCD this is $S U(2)_{L} \times S U(2)_{R} \sim S O(4)$. Since only the vector $S U(2)_{V} \sim S O(3)$ subgroup is realized in the spectrum, chiral symmetry must be spontaneously broken, leading to the appearance of three pseudo-Goldstone bosons that parametrize the coset space $S O(4) / S O(3) \sim S^{3}$, a 3 -sphere. Chiral symmetry is explicitly broken not only by the quark masses but also by electromagnetic and weaker interactions. Identifying the pseudo-Goldstone bosons as the three pions, we can write the interactions in the "..." of Eq. (2) so that $S U(2)_{L} \times S U(2)_{R}$ is broken just as in the underlying theory. For example, for the chirally symmetric pion kinetic term,

$$
\left[\left(\partial_{0} \boldsymbol{\pi}\right)^{2}-(\vec{\nabla} \boldsymbol{\pi})^{2}\right] \rightarrow\left[\left(\partial_{0} \boldsymbol{\pi}\right)^{2}-(\vec{\nabla} \boldsymbol{\pi})^{2}\right]\left(1-\frac{\boldsymbol{\pi}^{2}}{2 f^{2}}+\ldots\right)
$$

where $f$ is the bare pion decay constant. Equation (4) now gives, instead of Eq. (3),

$$
\begin{gathered}
m_{\pi}^{2}=\mathcal{O}\left(\hat{m} M_{\mathrm{QCD}}\right), \quad\left|\delta m_{\pi}^{2}\right|=\mathcal{O}\left(\alpha_{e} M_{\mathrm{QCD}}^{2} / 4 \pi\right), \\
m_{N}=\mathcal{O}\left(M_{\mathrm{QCD}}\right), \quad\left|\delta m_{N}\right|=\mathcal{O}\left(\epsilon \hat{m}, \alpha_{e} M_{\mathrm{QCD}} / 4 \pi\right), \\
f_{\pi}=\mathcal{O}\left(M_{\mathrm{QCD}} / 4 \pi\right), \quad\left|g_{A}\right|=\mathcal{O}(1) .
\end{gathered}
$$

These estimates work within a factor of $\sim 2$, yielding $m_{\pi}^{2} \sim(70 \mathrm{MeV})^{2}, \delta m_{\pi}^{2} \sim(30 \mathrm{MeV})^{2}$, $m_{N} \sim 1 \mathrm{GeV}, \delta m_{N} \sim 1 \mathrm{MeV}$, and $f_{\pi} \sim 80 \mathrm{MeV}$. The naturalness problems of plain dimensional analysis have been explained away by factors of $4 \pi$ (in the case of $g_{A}$ ) and, additionally, by approximate chiral symmetry (for $m_{\pi}^{2}, \delta m_{\pi}^{2}$, and $\delta m_{N}$ ).

This is a case where we could infer the existence of an approximate symmetry from a pattern of violation of naturalness, but it is not the only one. In another beautiful example, the magnitudes of lepton masses yield clues about the SM gauge structure [24]. Unfortunately, similar paths emerging from the smallness of the QCD vacuum angle, the Higgs mass parameter, and the cosmological constant are yet to be confirmed experimentally. 
What is the basis for NDA? The various elements that enter Feynman diagrams constructed from the Lagrangian (5) are

$$
\begin{aligned}
\text { loop integral } & \sim(4 \pi)^{-2} Q^{4}, \\
\text { fermion, boson propagator } & \sim Q^{-1}, Q^{-2}, \\
\text { vertex } & \sim(4 \pi)^{N-2} M_{\mathrm{hi}}^{4-D} c_{D N}^{\mathrm{red}} Q^{d},
\end{aligned}
$$

in terms of the typical external momentum $Q \sim M_{\mathrm{lo}}$. In a diagram with $L$ loops, these rules and standard graph identities can be used to show that the contribution to $c_{D N}^{\mathrm{red}}$ is $\mathcal{O}\left(\Lambda^{2 L} / M_{\mathrm{hi}}^{2 L}\right)$ times the product of reduced couplings from the various vertices. If all the reduced couplings are $\mathcal{O}(1)$, then $\Lambda \rightarrow M_{\text {hi }}$ implies $c_{D N}^{\text {red }}=\mathcal{O}(1)$. NDA is self-consistent in perturbation theory [5].

As a specific example, consider pion-pion scattering [5]. Schematically, the tree amplitude is

$$
T_{\pi \pi}^{(L=0)}(Q ; \Lambda) \sim \frac{Q^{2}}{f^{2}(\Lambda)}\left[1+c_{44}^{\mathrm{red}}(\Lambda) \frac{Q^{2}}{M_{\mathrm{hi}}^{2}}+\ldots\right],
$$

where the first term comes from the $b=4$ interactions arising from chiral symmetry (for $d=2$, see Eq. (6) ), while the second term is from $b=4$ interactions in the "..." of Eq. (2). Now, a generic one-loop diagram where the two vertices stem from the former interaction contains

$$
T_{\pi \pi}^{(L=1)}(Q ; \Lambda) \sim \frac{Q^{2}}{(4 \pi)^{2} f^{4}(\Lambda)}\left[\Lambda^{2}+Q^{2} \ln \left(\Lambda / m_{\pi}\right)\right] .
$$

The most severe cutoff dependence is removed by the first term in Eq. (11),

$$
f_{\pi}^{2} \sim f^{2}(\Lambda)+\frac{\Lambda^{2}}{(4 \pi)^{2}}+\ldots
$$

The replacement $\Lambda \rightarrow M_{\mathrm{QCD}}$ leads to the estimate for $f_{\pi}$ in Eq. (7). The $\ln \Lambda$ can then be absorbed by the second term in Eq. (11).

The situation is analogous for the Higgs mass,

$$
m_{H}^{2} \sim m^{2}(\Lambda)+c_{2} \Lambda^{2} /(4 \pi)^{2}+c_{0} m^{2}(\Lambda) \ln (\Lambda / m(\Lambda))+\ldots
$$

where $c_{0,2}$ are combinations of dimensionless coupling constants, which leads to the expectation $m_{H}=\mathcal{O}\left(M_{\mathrm{BSM}} / 4 \pi\right)$ in terms of the SM breakdown scale $M_{\mathrm{BSM}}$. Contrast this with the average pion mass, where only the $\ln \Lambda$ appears thanks to chiral symmetry [36, 37, 38]. Electromagnetic interactions break isospin and $\delta m_{\pi}^{2}$ does receive a $\Lambda^{2}$ contribution proportional to $\alpha_{e} / 4 \pi$. Again $\Lambda \rightarrow M_{\mathrm{QCD}}$ leads to the successful estimate (17). This example is in fact often invoked (for example, Refs. [22, 23]) in connection to the Higgs mass.

We have seen how NDA provides a benchmark against which to measure unnaturalness. From a reductionist perspective, the hope is that deviations will give clues about the underlying theory. From the point of view of emergence, in contrast, NDA offers a basis 
for the organization of interactions in the EFT itself, or power counting. In order to make predictions we need an amplitude at $Q \sim M_{\text {lo }}$ to be expressed in rough form as

$$
T\left(Q \sim M_{\mathrm{lo}}\right) \sim \mathcal{N} \sum_{\nu=0}^{\infty}\left(\frac{Q}{M_{\mathrm{hi}}}\right)^{\nu} F_{\nu}\left(Q / M_{\mathrm{lo}}\right),
$$

where $\nu$ is a counting index, $\mathcal{N}$ is a process-specific normalization, and $F_{\nu}\left(Q / M_{\mathrm{lo}}\right)$ is a combination of LECs and non-analytic functions stemming from loop integrals. The most important terms - the leading order $(\mathrm{LO})$ - have $\nu=0$, first corrections - next-toleading order (NLO) - have $\nu=1$, and so on.

In the absence of any small parameters, i.e., when all $c_{D N}^{\text {red }}=\mathcal{O}(1)$, the low-energy scale is $M_{\mathrm{lo}}=\mathcal{O}\left(M_{\mathrm{hi}} / 4 \pi\right)$. A diagram with $B(F)$ external boson (fermion) lines, $L$ loops, and $V_{i}$ vertices involving $d_{i}$ derivatives and $f_{i}$ fermion fields will then contribute at [2]

$$
\nu=2 L+\sum_{i} V_{i}\left(d_{i}+f_{i} / 2-2\right),
$$

while

$$
\mathcal{N} \sim\left(\frac{M_{\mathrm{hi}}}{4 \pi}\right)^{4-3 F / 2-B} .
$$

The factor of $2 L$ in Eq. (16) expresses the perturbative character of the amplitude. For example, not only are the higher-derivative terms suppressed in Eq. (11), but also the non-analytic part of the loop (12) can be treated perturbatively at $\mathrm{N}^{2} \mathrm{LO}$.

When an approximate symmetry leads to some $c_{D N}^{\text {red }} \ll 1$, they should be compared with $M_{\mathrm{lo}} / M_{\mathrm{hi}}=\mathcal{O}\left((4 \pi)^{-1}\right)$ so as to improve the power counting (16). Again $\chi$ EFT provides an explicit illustration. Since $\hat{m}^{\text {red }}=\mathcal{O}\left(m_{\pi}^{2} / M_{\mathrm{QCD}}^{2}\right)$, the effect of explicit chiral-symmetry breaking from quark masses is comparable to that of a derivative (Eq. (5)) for $Q \sim m_{\pi}$.

In the physical world $m_{\pi}$ is not very different from $f_{\pi}$, so we can for simplicity lump them together into $M_{\mathrm{lo}}$. The net effect on the power counting is that we can keep Eq. (16) as long as $d_{i}$ now counts powers of $m_{\pi}$ as well [2]. Similar steps can be taken for $(\epsilon \hat{m})^{\text {red }}$ and $e^{\text {red }}$.

But what if one or more of the LECs are unnatural after the existing symmetries are accounted for? The obvious guess is that an unknown symmetry is at play, in which case several LECs are likely affected. From the EFT perspective, the task is to include the unnatural LEC(s) in the power counting and go on with life. (If we keep having to repeat this procedure we might be seeing signs of a previously unknown low-energy degree of freedom, which again can be incorporated in the EFT, and a new power counting must be devised. The do-loop starts again.) We now look at how these ideas play out in nuclear physics.

\section{$3 \quad$ Nuclear unnaturalness?}

Is nuclear physics unnatural? In this section I discuss some of the evidence that suggests the answer might be "yes". I will argue that a small scale enters at the few-nucleon level, which could then spread through heavier systems. 
Before anything, though, we need to realize that NDA in the above form need not apply. As it stands, NDA has provided a successful basis for $\chi \mathrm{EFT}$ in processes with $Q \sim m_{\pi}$ where at most one nucleon is present $(A \leq 1)$ [39]. It is tempting to use it as a basis to study reactions and structure of systems with $A \geq 2$, but this attempt fails from the get-go. The reason lies on the existence in processes involving only heavy stable particles of "reducible" diagrams containing intermediate states devoid of light particles:

- The argument for NDA discussed above attributes $Q^{-1}$ to a fermion propagator, Eq. (9). This is a good estimate for a relativistic fermion, or for a single fermion of mass $m_{\psi} \gtrsim M_{\mathrm{hi}}$ interacting with an external relativistic boson. In both cases, the energy of the fermion in an intermediate state is typically $\mathcal{O}(Q)$. For a relativistic fermion, the 3-momentum is comparable. In the nonrelativistic case, the recoil is only $\mathcal{O}\left(Q^{2} / 2 m_{\psi}\right)$ and can be treated perturbatively, leaving the fermion as static at LO. However, if recoil were neglected in reducible diagrams, there would be infrared (IR) divergences. When recoil is retained, there is an IR enhancement $\mathcal{O}\left(m_{\psi} / Q\right)$ compared to the loops considered in the original NDA [10, 11].

- In integrals involving relativistic propagators, the typical factor resulting from angular integration is the $(4 \pi)^{-2}$ in Eq. (8) . For integrals where one picks the pole from a heavy particle propagator, though, there is usually an extra factor of $4 \pi$ coming from the contour integration over the magnitude of the 3-momentum [12, 13, 14, 15].

The net effect is to replace Eqs. (8) and (9) with

$$
\begin{aligned}
\text { reducible loop integral } & \sim\left(4 \pi m_{\psi}\right)^{-1} Q^{5}, \\
\text { fermion propagator } & \sim m_{\psi} Q^{-2} .
\end{aligned}
$$

A modified form of NDA results if we use these rules with the same rationale as before: we find the regulator cutoff dependence of an arbitrary loop and use $\Lambda \rightarrow M_{\text {hi }}$ to estimate the magnitude of the contribution from the related LECs to an observable.

The consequences for naturalness can be seen most easily in Pionless EFT ( $\star$ EFT) [3], a simpler nuclear EFT than $\chi$ EFT, where $M_{\text {hi }} \sim m_{\pi}$. Pions are integrated out, their effects being entirely encoded through LECs. If, for simplicity of notation, I leave the various spin-isospin factors implicit, the effective Lagrangian can be written as

$$
\begin{aligned}
\mathcal{L}_{\text {\#EFT }}= & N^{\dagger}\left(i \partial_{0}+\frac{\vec{\nabla}^{2}}{2 m_{N}}\right) N-\frac{2 \pi}{m_{N}}\left\{C_{0}\left(N^{\dagger} N\right)^{2}+C_{2}\left[\left(N^{\dagger} N\right)\left(N^{\dagger} \vec{\nabla}^{2} N\right)+\text { H.c. }\right]\right\} \\
& -\frac{(4 \pi)^{2}}{6 m_{N}} D_{0}\left(N^{\dagger} N\right)^{3}-\frac{(4 \pi)^{3}}{8 m_{N}} E_{0}\left(N^{\dagger} N\right)^{4}+\ldots,
\end{aligned}
$$

where $C_{0}, C_{2}, D_{0}$, and $E_{0}$ are LECs. Again, the “..." represent terms with more fields and derivatives. For a pedagogical introduction to $九 \mathrm{EFT}$, see Ref. [40].

Life is easier in $九 \mathrm{EFT}$ because all loops are reducible. In a case like this, we can replace Eq. (41) with

$$
c_{D N}=\mathcal{O}\left((4 \pi)^{N / 2-1} m_{\psi}^{-1} M_{\mathrm{hi}}^{5-D} c_{D N}^{\mathrm{red}}\right)
$$


Eq. (5) with

$$
\mathcal{L}_{\mathrm{EFT}} \sim(4 \pi)^{-1} m_{\psi}^{-1} M_{\mathrm{hi}}^{5} c_{D N}^{\mathrm{red}}\left(\frac{\partial}{M_{\mathrm{hi}}}\right)^{d}\left(\frac{4 \pi \bar{\psi} \psi}{M_{\mathrm{hi}}^{3}}\right)^{f / 2}
$$

and Eq. (10) with

$$
\text { vertex } \sim(4 \pi)^{N / 2-1} m_{\psi}^{-1} M_{\mathrm{hi}}^{5-D} c_{D N}^{\mathrm{red}} Q^{d},
$$

where again $c_{D N}^{\text {red }}$ is built from the reduced couplings in the underlying theory. Explicitly, in the absence of approximate symmetries,

$$
\left|C_{n}\right|=\mathcal{O}\left(M_{\mathrm{hi}}^{-(1+n)}\right), \quad\left|D_{n}\right|=\mathcal{O}\left(M_{\mathrm{hi}}^{-(4+n)}\right), \quad\left|E_{n}\right|=\mathcal{O}\left(M_{\mathrm{hi}}^{-(7+n)}\right),
$$

with the usual understanding that this represents the expected contribution of the corresponding interactions to observables after renormalization.

As an example, let us consider the two-nucleon system in a specific isospin channel, as we did pion-pion scattering earlier. The on-shell tree-level amplitude is

$$
T_{N N}^{(L=0)}(k ; \Lambda)=-\frac{4 \pi}{m_{N}}\left(C_{0}(\Lambda)-k^{2} C_{2}(\Lambda)+\ldots\right),
$$

where $k=\sqrt{m_{N} E}$ in terms of the center-of-mass energy $E$. The one-loop diagram which involves two successive contact interactions with the LEC $C_{0}$ gives

$$
T_{N N}^{(L=1)}(k ; \Lambda)=\frac{4 \pi}{m_{N}} C_{0}^{2}(\Lambda)\left(\theta_{1} \Lambda+i k+\theta_{-1} \frac{k^{2}}{\Lambda}+\ldots\right)
$$

where $\theta_{1-2 n}, n=0,1, \ldots$, are numbers that depend on the specific form of the (non-local) regulator [15]. With this type of regulator, the two-loop diagram with three $C_{0}$ s factorizes. Including this diagram, the amplitude becomes

$$
T_{N N}=\frac{4 \pi}{m_{N}} a_{2}\left[1-i k a_{2}-k^{2} a_{2}^{2}\left(1-\frac{r_{2}}{2 a_{2}}\right)+\ldots\right]
$$

after we define the inverse scattering length and the effective range, respectively

$$
a_{2}^{-1}=C_{0}^{-1}(\Lambda)+\theta_{1} \Lambda+\ldots, \quad r_{2}=-2 C_{0}^{-2}(\Lambda) C_{2}(\Lambda)-\theta_{-1} \Lambda^{-1}+\ldots
$$

Modified NDA translates into $\left|a_{2}^{-1}\right| \sim\left|r_{2}^{-1}\right|=\mathcal{O}\left(M_{\mathrm{hi}}\right)$. The suppression of the nonanalytic term in Eq. (26) by one power of $Q / M_{\mathrm{hi}}$ is different than the suppression by $\left(Q / M_{\mathrm{QCD}}\right)^{2}$ one finds when $A \leq 1$ (cf. Eq. (12)), but the effective-range correction is of relative $\mathcal{O}\left(Q^{2} / M_{\text {hi }}^{2}\right)$, as might have been expected from the two powers of $k$. One can repeat the argument for more-derivative operators and higher effective-range expansion parameters [15], recovering Eq. (24) for $C_{n}$.

To confirm this naturalness expectation we can consider as underlying theory an arbitrary potential of range $R \equiv M_{\mathrm{hi}}^{-1}$. Take, say, a three-dimensional spherical well [15, 41] with dimensionless depth $\alpha$,

$$
V(\vec{r})=-\frac{\alpha^{2}}{m R^{2}} \theta(R-r)
$$


Solving the Schrödinger equation for the $S$ wave and expanding the corresponding $T$ matrix in powers of $k R$, one finds the effective range parameters

$$
a_{2}=R\left(1-\frac{\tan \alpha}{\alpha}\right), \quad r_{2}=R\left(1-\frac{R}{\alpha^{2} a_{2}}-\frac{R^{2}}{3 a_{2}^{2}}\right), \quad \ldots
$$

For generic values of $\alpha$, we see that $\left|a_{2}\right| \sim\left|r_{2}\right| \sim \ldots \sim R$ as given by modified NDA. A plot of $a_{2} / R$ and $r_{2} / R$ as functions of $\alpha$ [41] shows that indeed these are the most common values. One way to quantify this is to assume equal probability for $\alpha$, which translates into a probability distribution for $a_{2} / R$ [41,

$$
p\left(a_{2} / R\right)=(\pi \alpha)^{-1}\left[\left(a_{2} / R-1\right)^{2}+\alpha^{-2}\right]^{-1}
$$

a plot of which shows a prominent peak at the natural value $a_{2}=R$.

Natural contact interactions with size (24) cannot generate bound states, as they are nonperturbative only for momenta comparable to the breakdown scale. That is a problem for nuclear physics, considering that empirically the two-nucleon amplitude has shallow poles in both $S$ channels: an isospin-singlet (triplet) $I=0(I=1)$ bound (virtual) state with imaginary momentum $i \kappa_{20}\left(i \kappa_{21}\right)$, where $\kappa_{20} \simeq a_{20}^{-1} \simeq 45 \mathrm{MeV}\left(\kappa_{21} \simeq a_{21}^{-1} \simeq-8\right.$ $\mathrm{MeV})$. Both binding momenta, especially $\kappa_{21}$, are unnatural compared with the expectation from modified NDA, $\left|a_{2 I}^{-1}\right|=\mathcal{O}\left(m_{\pi}\right)$.

This unnaturalness could be a reflection of fine tuning in the underlying theory. Again, the simple toy model (29) provides an example [15]: when $\alpha \simeq(2 n+1) \pi / 2 \equiv \alpha_{\mathrm{cn}}$ with $n \geq 0$ an integer, we have $\left|a_{2}\right| \simeq R\left|\alpha_{\mathrm{cn}}\left(\alpha-\alpha_{\mathrm{cn}}\right)\right|^{-1} \simeq\left|\kappa_{2}^{-1}\right| \gg R$ while still $\left|r_{2}\right| \sim \ldots \sim R$. For $\alpha$ just below $\alpha_{\mathrm{c} n}$, there is a shallow virtual state. As the attraction increases past $\alpha_{\mathrm{c} n}$, a shallow bound state appears. One can think of $a_{2}$ as the size of the bound state, which in quantum (in contrast to classical) mechanics can exceed the range of the potential 6 . This example evokes a real-life instance of fine tuning in atomic physics - a Feshbach resonance [42]. Some atomic systems consist of two coupled channels with different spin alignments and thresholds. Variation of an external magnetic field can bring a bound state in the closed channel to the open-channel threshold. The scattering length in the lower, open channel becomes arbitrarily large as the critical value of the magnetic field is approached. The knob that controls the magnetic field replaces $\alpha$.

Regardless of the reason for the appearance of shallow $S$-wave poles in the $T$ matrix, ÆEFT can describe them if after renormalization

$$
\left|C_{n}\right|=\mathcal{O}\left(\left|\kappa_{2}^{-(1+n / 2)}\right| M_{\mathrm{hi}}^{-n / 2}\right)
$$

with $\left|\kappa_{2}\right| \ll M_{\text {hi }}$. For $M_{\text {hi }} \gtrsim|k| \gtrsim\left|\kappa_{2}\right|$, all diagrams made out of $C_{0}$ are comparable and must be resummed. With a non-local regulator this can be done analytically, resulting in

\footnotetext{
${ }^{6}$ Note that this is not the only possible fine tuning in this toy model. One can also make $-r_{0} / R$ large by fine tuning $a_{2} / R$ to be small, that is, dialing a zero of the amplitude to the threshold region. The low-energy EFT for this situation is a Pionless EFT with a different scaling of $C_{n}$ [15] than discussed in the following.
} 
the LO amplitude

$$
T_{N N}^{(0)}(k ; \Lambda)=\frac{4 \pi}{m}\left(\kappa_{2}+i k\right)^{-1}
$$

where

$$
\kappa_{2}=C_{0}^{-1}(\Lambda)+\theta_{1} \Lambda
$$

and I dropped terms that can be made arbitrarily small for arbitrarily large $\Lambda$. Unnaturalness comes from the failure of the replacement $\Lambda \rightarrow M_{\mathrm{hi}}$ as an estimate for $\kappa_{2}$.

A fully systematic description emerges of the low-energy two-nucleon system [3], when the subleading interactions are treated in distorted-wave perturbation theory to ensure renormalization [12, 13, 14, 15. Having incorporated the unnaturalness at LO, we might expect that corrections will contain no additional failure of naturalness, so that subleading LECs can be estimated from loop diagrams by $\Lambda \rightarrow M_{\mathrm{hi}}$. For example, the $\Lambda^{-1}$ term in Eq. (28) leads to $C_{2}$ at NLO as given by Eq. (32) - this is one order down the expansion, in contrast to (modified) NDA. Equivalently, $\left|r_{2}\right|=\mathcal{O}\left(M_{\mathrm{hi}}^{-1}\right)$ just as expected from naturalness. The argument continues at higher order.

For $|k| \ll\left|\kappa_{2}\right|$ we can Taylor-expand the denominator of Eq. (33) to obtain Eq. (27). As far as the first two terms of the latter are concerned, it seems as if we are back to the naturalness case with $M_{\mathrm{hi}}=\kappa_{2}$, but effective-range parameters other than the scattering length would look small. A better quantum-field theoretical example to replace a nonrelativistic potential as a toy underlying theory is a Pionless EFT for two coupled channels [43, 44, which models a Feshbach resonance. When all entries of the $2 \times 2$ matrix that replaces $C_{0}$ have about the same magnitude $\kappa_{R}^{-1}$ and the channel thresholds differ in energy by $\Delta E \sim \kappa_{R}^{2} / 2 \mu$ (where $\mu$ is the reduced mass), one generally finds $\left|a_{2}\right| \sim\left|r_{2}\right| \sim \kappa_{R}^{-1}$ in the open channel — the natural situation. By dialing $\kappa_{R}^{-1}$ against a combination of entries of the $2 \times 2$ coupling matrix, one can produce $\left|a_{2}\right| \gg \kappa_{R}^{-1}$ while $r_{2} \sim-\kappa_{R}^{-1}$ - the fine-tuned scenario.

Now that we have an EFT that accounts for shallow two-nucleon states, we may ask what the consequences for larger nuclei are. The answer is, lots of surprises. The first surprise was probably the observation by Thomas [45] of a "collapse" of the $A=3$ system. With the LO two-body interactions, the ground-state binding energy is $B_{3} \propto \Lambda^{2} / m_{N}$ for $\Lambda \gg\left|\kappa_{2}\right|$ and, as $\Lambda$ increases, excited bound states emerge and collapse as well. The half-off-shell amplitude for scattering of a particle on the two-body bound state displays a bizarre behavior: it oscillates as a function of the off-shell momentum with a phase that depends on $\ln \Lambda$ [46, 47, 48]. Small cutoff variations result in large changes at low momentum, a regulator dependence that indicates that the $A=3$ system is not renormalizable with only $C_{0}$ interactions. The same conclusion holds for bosons, which also suffer from the absence of the Pauli exclusion principle.

The second surprise is that, since two-body interactions with more derivatives are small within the range of the EFT, the appropriate counterterm must be a three-body force. And an unexpected one at that. Indeed, the $D_{0}$ term in Eq. (20) can exactly 
counterbalance the cutoff variation if [46, 47, 48,

$$
D_{0}(\Lambda) \propto \frac{1}{\Lambda^{4}} \frac{\sin \left(s_{0} \ln \left(\Lambda / \Lambda_{\star}\right)-\arctan s_{0}^{-1}\right)}{\sin \left(s_{0} \ln \left(\Lambda / \Lambda_{\star}\right)+\arctan s_{0}^{-1}\right)}\left(1+\mathcal{O}\left(\kappa_{2} / \Lambda\right)\right),
$$

where $s_{0} \simeq 1.00624$ and $\Lambda_{\star}$ is a physical parameter. Once one low-momentum datum is reproduced by a choice of $\Lambda_{\star}$, the phase of the half-off-shell scattering amplitude is fixed and all other low-momentum observables attain finite values as $\Lambda$ increases. This is true, in particular, of bound states. Instead of the periodic emergence of bound states at zero energy before renormalization, after renormalization one observes the periodic emergence of deeper bound states, which achieve finite binding energies as $\Lambda$ increases. The bound states that appear once $\Lambda \gtrsim M_{\mathrm{hi}}$ are unphysical because they are beyond the range of validity of the EFT.

Unlike the two-derivative two-body force, the two-derivative three-body force with LEC $D_{2}$ (to be found in the "..." of Eq. (20)) appears at $\mathrm{N}^{2} \mathrm{LO}$ [47, 49, 50, 51, 52]. Since $D_{0}$ is an LO interaction, the three-body LECs effectively scale as

$$
\left|D_{0}\right|=\mathcal{O}\left(M_{\mathrm{lo}}^{-4}\right), \quad\left|D_{2}\right|=\mathcal{O}\left(M_{\mathrm{lo}}^{-4} M_{\mathrm{hi}}^{-2}\right) .
$$

This is very different from what is expected from modified NDA with natural-sized $C_{0}$. Consider the two-loop three-body diagram with four successive $C_{0}$ interactions involving each time a different pair of particles. Under the rules (18) and (19), it generates a $\ln \Lambda$ dependence, whose coefficient leads to the scaling of $D_{0}$ in Eq. (24). Conversely, an unnatural $C_{0}$ induces an unnatural $D_{0}$. However, while this diagram for unnatural $C_{0}$ suggests that $M_{\mathrm{lo}}$ is determined by $\kappa_{2}$, the nonperturbative renormalization that leads to Eq. (35) means the three-body energies are fixed by $\Lambda_{\star} . M_{\mathrm{lo}}$ is set by $\kappa_{3}$.

There is no relative renormalization enhancement of higher-body forces at LO, as the calculations of Refs. [53, 54, 55, 56, 57, 58, 59, 60] found convergence in $A=4,5,6$ binding energies as $\Lambda$ increases. An enhancement takes place at NLO, though, so that a new scale appears through a four-body force 61]

$$
\left|E_{0}\right|=\mathcal{O}\left(M_{\mathrm{lo}}^{-6} M_{\mathrm{hi}}^{-1}\right) \text {. }
$$

Once this force is accounted for, no more-body forces are needed for NLO renormalization in larger systems.

Therefore, at LO there is a single parameter $\Lambda_{\star}$ not determined by $A=2$ physics. Not every $A \geq 3$ observable is sensitive to this parameter. For example, neutron-deuteron (nd) scattering in the spin-3/2 channel, where the two neutron spins are aligned, can be predicted to a very good accuracy from $A=2$ physics [62, 63, 64]. But correlations should exist through $\Lambda_{\star}$ among $A \geq 3$ observables not affected by the exclusion principle. The classic example is the Phillips line [65] on the plane spanned by the triton binding energy and the spin-1/2 nd scattering length. This correlation was first discovered empirically with points representing various phenomenological potentials, which describe two-nucleon data up to relatively high momenta. In the EFT, this correlation is produced as $\Lambda_{\star}$ is 
varied [46, 47, 48]. The EFT line lies close not only to the experimental point but also to the empirical line, meaning that the many parameters of the various phenomenological potentials amount to a single relevant parameter $\Lambda_{\star}$. Similarly, $A \geq 4$ ground-state binding energies are correlated with the $A=3$ ground-state energy [53, 54, 59] in a Tjon [66, 67] and generalized Tjon [68, 69] lines.

ÆEFT bags many other successes for light nuclei [3]. It is not currently known how far in $A \not \mathrm{EFT}$ can accommodate the growing nuclear binding energies. But within its regime of validity we should be able to derive nuclear properties with the contact interactions in Eq. (20) . The small scales they contain will contaminate heavier nuclei and lead to anomalously small energies or energy gaps. The application of $九 \mathrm{EFT}$ to $A \geq 5$ nuclei is in its infancy. The first indication [55, 60, 70] is that at LO a gas of nucleons and alpha particles results. Whether subleading corrections will lead to the relatively small binding (relative to these nucleon-alpha thresholds) is an open question. We might conjecture that, in addition to purely accidental near coincidences among the large number of nuclear excited states and thresholds, unnatural scales in heavier nuclei trace back to those in light nuclei.

\section{Unraveling unnaturalness}

Is nuclear physics technically unnatural, though? In this section I discuss some of the evidence that suggests the answer might be "no". I will argue that there is an approximate symmetry that ensures that a small scale enters nuclear physics.

In the scale of the triton binding binding momentum $\kappa_{3} \simeq 70 \mathrm{MeV}$, the ${ }^{1} S_{0}$ two-nucleon pole is very shallow, and even the deuteron binding momentum is somewhat small. This suggests that the unitarity limit $\kappa_{2} \rightarrow 0$ in Eq. (33) might be a good approximation for the physics of the ground states of larger nuclei [16, 17, 18, 19, 20]. (See also Refs. [71, 172, for a related approach.) In this limit, which is a non-trivial fixed point of the renormalization group (RG) [11, the LO two-nucleon amplitude (33) has only the unitarity term $i k$, with no dimensionful parameter.

The vanishing of two-body binding energies in the unitarity limit is a reflection of scale invariance. Under a change of scales [73] with parameter $\alpha>0$,

$$
r \rightarrow \alpha r, \quad t / m_{\psi} \rightarrow \alpha^{2} t / m_{\psi}, \quad \Lambda \rightarrow \alpha^{-1} \Lambda, \quad \psi \rightarrow \alpha^{-3 / 2} \psi,
$$

the nucleon kinetic and $C_{0}$ terms in Eq. (20) are invariant, but only when $\kappa_{2} \rightarrow 0$ in Eq. (34). Under a scale change, $m E \rightarrow \alpha^{-2} m E$ but in the unitarity limit there is no two-body scale, so $B_{2}$ must vanish. In this limit the $A=2$ system is also conformally invariant [74].

Beyond the two-body system, scale (as well as conformal) invariance is "anomalously" broken by the dimensionful parameter $\Lambda_{\star}$. The latter arises from renormalization, even though at unitarity we start without any scale, so we could call it dimensional transmutation. The dependence of $D_{0}$ on $\Lambda$ in Eq. (35) reveals an RG limit cycle [46, 47, 48, As a consequence, all LO terms in Eq. (20) are invariant under a transformation (38), but only for discrete values

$$
\alpha_{l}=e^{l \pi / s_{0}} \simeq(22.7)^{l}
$$


with $l$ an integer. The limit cycle engenders discrete scale invariance (DSI) [75] which is present for all $A$ within the range of $九 \mathrm{EFT}$.

Since it is the only dimensionful parameter at two-body unitarity, $\Lambda_{\star}$ sets the scale for all $A \geq 3$ binding energies. For ground states, eliminating $\Lambda_{\star}$ translates into a universal form [76] for the correlations mentioned in the previous section,

$$
\frac{B_{A}}{A}=\xi_{A} \frac{B_{3}}{3} .
$$

The numbers $\xi_{A}$ are universal in the sense that they are the same for any type of unitary four-component fermion. The same relation holds for bosons with the same value for $\xi_{4}$ but different $\xi_{A \geq 5}$ on account of the absence of the exclusion principle. The value $\xi_{4} \simeq 3.46$ is well established [77]. For bosons we find at small $A$ [59]

$$
\xi_{A} \approx 3 A\left(1-\frac{2}{A}\right)^{2},
$$

while for large $A[76]$

$$
\xi_{A} \approx \xi_{\infty}\left(1-\frac{\eta}{A^{1 / 3}}+\ldots\right),
$$

with $\xi_{\infty} \simeq 90 \pm 10$ and $\eta \simeq 1.7 \pm 0.3$. Many bosons at unitarity thus approximately satisfy, like nuclei, the liquid-drop formula and can be thought of forming a quantum liquid. The behavior is similar to that of ${ }^{4} \mathrm{He}$ atomic clusters [78].

For nucleons, not much is known. When corrections linear in $a_{2}^{-1}$ are included, $\xi_{4}$ becomes $\simeq 2.6$ [17]. This is very close to the experimental value $\xi_{4}^{\exp } \simeq 2.5$ obtained from the triton and alpha binding energies. A full NLO calculation involves the four-body force with LEC $E_{0}$ [61], which can be adjusted to reproduce $\xi_{4}^{\exp }$. A small number $(A=8)$ of unitary four-component fermions tends to cluster into alpha-like objects [79], as observed in ${ }^{8}$ Be. The behavior for other $A$ is virgin territory, even at LO.

DSI has strong consequences also for excited states. For $A=3$ I write binding energies as

$$
B_{3 ; n}=\beta_{3 ; n} B_{3},
$$

with $n \geq 0$ and $\beta_{3 ; 0}=1$. The $m E \rightarrow \alpha_{l}^{-2} m E$ transformation under a scale change can now support non-vanishing energies if

$$
\beta_{3 ; n}=e^{-2 n \pi / s_{0}} \text {. }
$$

This is Efimov's famous geometric tower [80, 81], which extends up to threshold. An extensive review of the properties of these states can be found in Ref. [41]. As twobody attraction decreases and $\kappa_{2}$ becomes negative, one observes the amazing quantum phenomenon of Borromean binding, where the three-body system shows bound states while no two-body bound state exists. If, instead, attraction increases and $\kappa_{2}$ becomes positive, three-body bound states disappear into the particle-dimer threshold. In either case, only a few of these states are physical. For atomic ${ }^{4} \mathrm{He}$, both ground 82 and firstexcited [83] states have been detected. For nucleons, only the ground state (triton/helion) 
is observed, but there is a virtual state in $n d$ scattering that becomes the triton excited state as $\kappa_{20}$ is decreased [84].

For $A=4$, there are two states for each Efimov state [56], one 1.002 more bound than the $A=3$ parent, the other $\simeq 4.6$ times deeper [77]. Remarkably, potential-model calculations [85, 86, 87, 88] show that this doubling process continues with increasing number of bosons. For a given $A$, there are $2^{(A-3)}$ interlocking towers of states. The replicating towers are a reflection of DSI, but the doubling, which has a topological interpretation [89], is of unclear origin. For $A \geq 5$ four-component fermions the pattern of towers is not known.

The lowest $A=4$ Efimov-state descendants have been spotted in atomic systems [90]. The first excited state of the alpha particle is close to the nucleon-triton threshold, another evidence that light nuclei are perturbatively close to unitarity [17]. This state can be thought of as a two-body, nucleon-triton "halo" state, since its separation energy is much smaller than the triton binding energy. One would have thought this situation could only arise from fine tuning! Yet, each top boson state in the doubling is automatically a halo state consisting of a boson and an $A-1$ boson cluster. It is possible that similar structures arise for five or more unitary four-component fermions, and indeed they are observed in "halo nuclei". The poster child is ${ }^{6} \mathrm{He}$, which is only $\simeq 1.04$ times more bound than the alpha particle and can be viewed as a three-body state of two neutrons and one alpha. It could thus be that even such an apparent fine tuning has an origin in DSI. Regardless of the cause, halo states can be described by an EFT, Halo EFT [91, 92, in a way that is completely parallel to $九 \mathrm{EFT}$ for nucleons, only with additional fields for tight clusters of nucleons such as the alpha particle.

\section{Unclear naturalness?}

I have emphasized that some notion of naturalness is necessary for power counting, which in turn is essential for EFT predictive power. In perturbative theories, naive dimensional analysis provides a reasonable definition of naturalness. I went on to show that a modification of NDA needed for heavy fermion systems implies that light nuclei are contaminated by a small scale. I linked this small scale to approximate discrete scale invariance, which, to the extent it holds, ensures the properties of heavier nuclei are tied to those of the threebody system. I even speculated, based on calculations for bosons, that some coincidences observed in nuclear physics could, perhaps, be generated automatically.

Still, there are some clouds on the horizon. First, due to the nonperturbative nature of nuclei, certain interactions in the effective Lagrangian (20) are enhanced in the limit DSI is exact, rather than suppressed as per 't Hooft's expectation. This is not necessarily a problem, as the introduction of auxiliary fields shows. To handle the shallow states we can introduce two "dimer" fields $D_{I}$ [93] and one "trimer" field $T$ [50]. Still leaving the 
two channels $I=0,1$ implicit,

$$
\begin{aligned}
\mathcal{L}_{\text {सEFT }}= & N^{\dagger}\left(i \partial_{0}+\frac{\vec{\nabla}^{2}}{2 m_{N}}\right) N \\
& +D^{\dagger}\left[-\Delta+\delta\left(i m_{N} \partial_{0}+\frac{\vec{\nabla}^{2}}{4}\right)\right] D-\sqrt{\frac{4 \pi}{m_{N}}}\left(N^{\dagger} N^{\dagger} D+\text { H.c. }\right) \\
& +T^{\dagger}\left[-\Omega+\omega\left(i m_{N} \partial_{0}+\frac{\vec{\nabla}^{2}}{6}+\frac{\kappa_{2}^{2}}{m_{N}}\right)\right] T-\sqrt{4 \pi}\left(N^{\dagger} D^{\dagger} T+\text { H.c. }\right) \\
& +4 \pi \Theta_{0} T^{\dagger} T N^{\dagger} N+\ldots,
\end{aligned}
$$

where $\Delta=C_{0}^{-1}, \delta=C_{0}^{-2} C_{2}, \Omega=C_{0}^{2} D_{0}^{-1}, \omega=C_{0}^{2} D_{0}^{-2} D_{2}, \Theta_{0}=C_{0}^{2} D_{0}^{-2} E_{0}$, etc. As the only two-body parameter with non-negative mass dimension $(+1)$, one might expect the scale-invariance suppression $\Delta^{\text {red }}=\mathcal{O}\left(\kappa_{2} / M_{\mathrm{hi}}\right)$ in the dimeron residual mass. It resembles the effect of symmetries on other masses - e.g. on the nucleon mass difference (7) that appears in the Lagrangian (2) - and it is indeed sufficient to convert the naturalness relation (24) into the near-unitarity expectation (32). For the three-body parameters with non-negative mass dimensions $(+2$ and 0$)$, we have to assume $\Omega^{\text {red }}=\mathcal{O}\left(M_{\mathrm{lo}}^{2} / M_{\mathrm{hi}}^{2}\right)=\omega^{\text {red }}$, which then yields the relations (36) and (37). The ratio $M_{\mathrm{lo}} / M_{\mathrm{hi}} \ll 1$ must somehow arise once $\kappa_{2} / M_{\mathrm{hi}} \ll 1$.

Second, it is unclear how the symmetry emerges in QCD or even $\chi \mathrm{EFT}$. The most important element of $\chi \mathrm{EFT}$ in nuclear physics is one-pion exchange (OPE). At $Q \sim m_{\pi}$, OPE has a magnitude [13, 14]

$$
\left|V_{\mathrm{OPE}}\right|=\mathcal{O}\left(4 \pi m_{N}^{-1} M_{N N}^{-1}\right), \quad M_{N N} \equiv \frac{16 \pi f_{\pi}^{2}}{g_{A}^{2} m_{N}} .
$$

While NDA implies $M_{N N}=\mathcal{O}\left(f_{\pi}\right)$, numerically $M_{N N} \simeq 290 \mathrm{MeV}$. For $Q \sim M_{N N}$, pions are nonperturbative. OPE is a singular potential in spin-triplet waves, and its nonperturbative renormalization requires a chirally symmetric LEC in each wave where OPE is attractive [94]. The solution of the Schrödinger equation oscillates with a wavelength determined by $M_{N N}$ while the LEC fixes the asymptotic phase [95]. Although OPE is not singular in the ${ }^{1} S_{0}$ channel, its interference with the chirally symmetric contact interaction demands an additional chiral-symmetry-breaking LEC for renormalization [96]. In both $S$-wave channels it is thus a combination of OPE and LEC that must enforce shallow two-nucleon poles. But $M_{N N}$ remains and scale invariance is apparently broken explicitly. Scale invariance must be an emergent symmetry for $Q \lesssim M_{N N}$, where pion exchange should be perturbative [13, 14]. In this case, DSI manifests itself just as in $\Varangle \mathrm{EFT}$. Unfortunately the perturbative-pion expansion breaks down already below $M_{N N}$, at least in the ${ }^{3} S_{1}$ and ${ }^{3} P_{0}$ channels [97, 98]. The convergence for ${ }^{1} S_{0}$ is also slow at best [99]. The numerical character of nonperturbative OPE calculations obscures the emergence of DSI. 
There is much to do, both in exploring the manifestations of DSI in larger nuclei and in understanding its emergence in QCD. Still, despite the current challenges in particle physics, a criterion of naturalness supplies an essential ingredient for nuclear EFTs.

\section{Acknowledgments}

I thank Matt Baumgart, Ozan Erdogan, and Jaber Balal Habashi for useful discussions. This material is based upon work supported in part by the U.S. Department of Energy, Office of Science, Office of Nuclear Physics, under award DE-FG02-04ER41338 and by the European Union Research and Innovation program Horizon 2020 under grant No. 654002.

\section{References}

[1] M. Dine, Ann. Rev. Nucl. Part. Sci. 65 (2015) 43.

[2] S. Weinberg, Physica A 96 (1979) 327.

[3] H.-W. Hammer, S. König, and U. van Kolck, arXiv:1906.12122 [nucl-th].

[4] R. Machleidt, Int. J. Mod. Phys. E 26 (2017) 1730005.

[5] A. Manohar and H. Georgi, Nucl. Phys. B 234 (1984) 189.

[6] H. Georgi and L. Randall, Nucl. Phys. B 276 (1986) 241.

[7] S. Weinberg, Phys. Rev. Lett. 63 (1989) 2333.

[8] H. Georgi, Phys. Lett. B 298 (1993) 187.

[9] U. van Kolck, arXiv:2003.06721 [nucl-th].

[10] S. Weinberg, Phys. Lett. B 251 (1990) 288.

[11] S. Weinberg, Nucl. Phys. B 363 (1991) 3.

[12] U. van Kolck, Lect. Notes Phys. 513 (1998) 62.

[13] D.B. Kaplan, M.J. Savage, and M.B. Wise, Phys. Lett. B 424 (1998) 390.

[14] D.B. Kaplan, M.J. Savage, and M.B. Wise, Nucl. Phys. B 534 (1998) 329.

[15] U. van Kolck, Nucl. Phys. A 645 (1999) 273.

[16] S. König, H.W. Grießhammer, H.-W. Hammer, and U. van Kolck, J. Phys. G 43 (2016) 055106. 
[17] S. König, H.W. Grießhammer, H.-W. Hammer, and U. van Kolck, Phys. Rev. Lett. 118 (2017) 202501.

[18] S. König, J. Phys. G 44 (2017) 064007.

[19] U. van Kolck, Few-Body Syst. 58 (2017) 112.

[20] S. König, arXiv:1910.12627 [nucl-th].

[21] P. Nelson, Am. Sci. 73 (1985) 60.

[22] G.F. Giudice, In *Kane, Gordon (ed.), Pierce, Aaron (ed.): Perspectives on LHC physics* 155-178, arXiv:0801.2562 [hep-ph]].

[23] A. Grinbaum, Found. Phys. 42 (2012) 615.

[24] J.D. Wells, Stud. Hist. Phil. Sci. B 49 (2015) 102.

[25] P. Williams, Stud. Hist. Phil. Sci. B 51 (2015) 82.

[26] S. Hossenfelder, arXiv:1801.02176 [physics.hist-ph].

[27] J. Bain, Found. Phys. 49 (2019) 898.

[28] J.D. Wells, Found. Phys. 49 (2019) 991.

[29] P. Williams, Found. Phys. 49 (2019) 1022.

[30] A. Borrelli and E. Castellani, Found. Phys. 49 (2019) 860.

[31] P.A.M. Dirac, Nature 139 (1937) 323.

[32] J. Gasser and H. Leutwyler, Annals Phys. 158 (1984) 142.

[33] M.J.G. Veltman, Acta Phys. Polon. B 12 (1981) 437.

[34] C.P. Burgess, arXiv:1309.4133 [hep-th].

[35] G. 't Hooft, NATO Sci. Ser. B 59 (1980) 135.

[36] J.M. Charap, Phys. Rev. D 2 (1970) 1554; Addendum: [Phys. Rev. D 3 (1971) 1998].

[37] J. Honerkamp and K. Meetz, Phys. Rev. D 3 (1971) 1996.

[38] I.S. Gerstein, R. Jackiw, S. Weinberg, and B.W. Lee, Phys. Rev. D 3 (1971) 2486.

[39] V. Bernard, Prog. Part. Nucl. Phys. 60 (2008) 82.

[40] U. van Kolck, arXiv:1902.03141 [nucl-th].

[41] E. Braaten and H.-W. Hammer, Phys. Rept. 428 (2006) 259. 
[42] C. Chin, R. Grimm, P. Julienne, and E. Tiesinga, Rev. Mod. Phys. 82 (2010) 1225.

[43] T.D. Cohen, B.A. Gelman, and U. van Kolck, Phys. Lett. B 588 (2004) 57.

[44] E. Braaten, M. Kusunoki, and D. Zhang, Annals Phys. 323 (2008) 1770.

[45] L.H. Thomas, Phys. Rev. 47 (1935) 903.

[46] P.F. Bedaque, H.-W. Hammer, and U. van Kolck, Phys. Rev. Lett. 82 (1999) 463.

[47] P.F. Bedaque, H.-W. Hammer, and U. van Kolck, Nucl. Phys. A 646 (1999) 444.

[48] P.F. Bedaque, H.-W. Hammer, and U. van Kolck, Nucl. Phys. A 676 (2000) 357.

[49] H.-W. Hammer and T. Mehen, Phys. Lett. B 516 (2001) 353.

[50] P.F. Bedaque, G. Rupak, H.W. Grießhammer, and H.-W. Hammer, Nucl. Phys. A 714 (2003) 589.

[51] L. Platter, C. Ji, and D.R. Phillips, Phys. Rev. A 79 (2009) 022702.

[52] C. Ji and D.R. Phillips, Few Body Syst. 54 (2013) 2317.

[53] L. Platter, H.-W. Hammer, and U.-G. Meißner, Phys. Rev. A 70 (2004) 052101.

[54] L. Platter, H.-W. Hammer, and U.-G. Meißner, Phys. Lett. B 607 (2005) 254.

[55] I. Stetcu, B.R. Barrett, and U. van Kolck, Phys. Lett. B 653 (2007) 358.

[56] H.-W. Hammer and L. Platter, Eur. Phys. J. A 32 (2007) 113.

[57] J. Kirscher, H.W. Grießhammer, D. Shukla, and H.M. Hofmann, Eur. Phys. J. A 44 (2010) 239.

[58] J. Kirscher, N. Barnea, D. Gazit, F. Pederiva, and U. van Kolck, Phys. Rev. C 92 (2015) 054002.

[59] B. Bazak, M. Eliyahu, and U. van Kolck, Phys. Rev. A 94 (2016) 052502.

[60] L. Contessi, A. Lovato, F. Pederiva, A. Roggero, J. Kirscher, and U. van Kolck, Phys. Lett. B 772 (2017) 839.

[61] B. Bazak, J. Kirscher, S. König, M. Pavón Valderrama, N. Barnea, and U. van Kolck, Phys. Rev. Lett. 122 (2019) 143001.

[62] P.F. Bedaque and U. van Kolck, Phys. Lett. B 428 (1998) 221.

[63] P.F. Bedaque, H.-W. Hammer, and U. van Kolck, Phys. Rev. C 58 (1998) R641.

[64] J. Vanasse, Phys. Rev. C 88 (2013) 044001. 
[65] A.C. Phillips, Nucl. Phys. A 107 (1968) 209.

[66] J.A. Tjon, Phys. Lett. 56B (1975) 217.

[67] S. Nakaichi, Y. Akaishi, H. Tanaka, and T.K. Lim, Phys. Lett. A 68 (1978) 36.

[68] S. Nakaichi, T.K. Lim, Y. Akaishi, and H. Tanaka, J. Chem. Phys. 71 (1979) 4430.

[69] T.K. Lim, S. Nakaichi, Y. Akaishi, and H. Tanaka, Phys. Rev. A 22 (1980) 28.

[70] A. Bansal, S. Binder, A. Ekström, G. Hagen, G.R. Jansen, and T. Papenbrock, Phys. Rev. C 98 (2018) 054301.

[71] A. Kievsky, M. Viviani, D. Logoteta, I. Bombaci, and L. Girlanda, Phys. Rev. Lett. 121 (2018) 072701.

[72] M. Gattobigio, A. Kievsky, and M. Viviani, Phys. Rev. C 100 (2019) 034004.

[73] C.R. Hagen, Phys. Rev. D 5 (1972) 377.

[74] T. Mehen, I.W. Stewart, and M.B. Wise, Phys. Lett. B 474 (2000) 145.

[75] D. Sornette, Phys. Rept. 297 (1998) 239.

[76] J. Carlson, S. Gandolfi, U. van Kolck, and S.A. Vitiello, Phys. Rev. Lett. 119 (2017) 223002.

[77] A. Deltuva, Phys. Rev. A 82 (2010) 040701.

[78] V.R. Pandharipande, J.G. Zabolitzky, S.C. Pieper, R.B. Wiringa, and U. Helmbrecht, Phys. Rev. Lett. 50 (1983) 1676.

[79] W.G. Dawkins, J. Carlson, U. van Kolck, and A. Gezerlis, arXiv:1908.04288 [condmat.quant-gas].

[80] V. Efimov, Phys. Lett. 33B (1970) 563.

[81] V.N. Efimov, Sov. J. Nucl. Phys. 12 (1971) 589.

[82] W. Schöllkopf and J.P. Toennies, J. Chem. Phys. 104 (1996) 1155.

[83] M. Kunitski et al., Science 348 (2015) 551.

[84] G. Rupak, A. Vaghani, R. Higa, and U. van Kolck, Phys. Lett. B 791 (2019) 414.

[85] J. von Stecher, J. Phys. B 43 (2010) 101002.

[86] M. Gattobigio, A. Kievsky, and M. Viviani, Phys. Rev. A 84 (2011) 052503.

[87] J. von Stecher, Phys. Rev. Lett. 107 (2011) 200402. 
[88] M. Gattobigio, A. Kievsky, and M. Viviani, Phys. Rev. A 86 (2012) 042513.

[89] Y. Horinouchi and M. Ueda, Phys. Rev. A 94 (2016) 050702.

[90] F. Ferlaino et al., Phys. Rev. Lett. 102 (2009) 140401.

[91] C.A. Bertulani, H.-W. Hammer, and U. van Kolck, Nucl. Phys. A 712 (2002) 37.

[92] P.F. Bedaque, H.-W. Hammer, and U. van Kolck, Phys. Lett. B 569 (2003) 159.

[93] D.B. Kaplan, Nucl. Phys. B 494 (1997) 471.

[94] A. Nogga, R.G.E. Timmermans, and U. van Kolck, Phys. Rev. C 72 (2005) 054006.

[95] S.R. Beane, P.F. Bedaque, L. Childress, A. Kryjevski, J. McGuire, and U. van Kolck, Phys. Rev. A 64 (2001) 042103.

[96] D.B. Kaplan, M.J. Savage, and M.B. Wise, Nucl. Phys. B 478 (1996) 629.

[97] S. Fleming, T. Mehen, and I.W. Stewart, Nucl. Phys. A 677 (2000) 313.

[98] D.B. Kaplan, arXiv:1905.07485 [nucl-th].

[99] S.R. Beane, P.F. Bedaque, M.J. Savage, and U. van Kolck, Nucl. Phys. A 700 (2002) 377. 